\title{
Non-eliminative Structuralism, Fregean Abstraction, and Non-rigid Structures
}

\author{
John Wigglesworth ${ }^{1}[$
}

Received: 22 December 2016 / Accepted: 8 December 2018 / Published online: 18 December 2018

(c) The Author(s) 2018

\begin{abstract}
Linnebo and Pettigrew (Philos Q 64:267-283, 2014) have recently developed a version of non-eliminative mathematical structuralism based on Fregean abstraction principles. They recognize that this version of structuralism is vulnerable to the well-known problem of non-rigid structures. This paper offers a solution to the problem for this version of structuralism. The solution involves expanding the languages used to describe mathematical structures. We then argue that this solution is philosophically acceptable to those who endorse mathematical structuralism based on Fregean abstraction principles.
\end{abstract}

Mathematical structuralists who think that abstract structures exist face a problem. The problem comes from the existence of non-rigid structures, structures that admit non-trivial automorphisms. A non-trivial automorphism is an isomorphism from a structure to itself that is not the identity mapping; under the automorphism, there is an object in the structure that is not mapped to itself. Non-trivial automorphisms create a problem for mathematical structuralism. Some versions of structuralism entail that objects in a structure with exactly the same properties are identical. Nontrivial automorphisms on a structure show that the structure has two distinct objects with the same properties. The structuralist is thus forced to identify objects that should be distinct. This is the problem of non-rigid structures.

The problem of non-rigid structures is a general problem threatening several versions of non-eliminative structuralism. It was originally developed in response to Stewart Shapiro's (1997) ante rem structuralism, most notably by Burgess (1999), Hellman (2001) and Keränen (2001, 2006). But as Linnebo and Pettigrew (2014) have shown, the problem also threatens other forms of non-eliminative structuralism. In particular, versions of structuralism based on abstraction principles are affected by the problem of non-rigid structures.

John Wigglesworth

john.wigglesworth@univie.ac.at

1 Institut für Philosophie, University of Vienna, Universitätsstrasse 7, 1010 Vienna, Austria 
This paper offers a solution to the problem of non-rigid structures for a version of non-eliminative structuralism based on Fregean abstraction principles. Section 1 describes mathematical structuralism in more detail. The particular kind of mathematical structuralism under consideration takes mathematical structures to be obtained through a process of Fregean abstraction, which is the focus of Sect. 2. Section 3 describes the non-rigidity problem for this version of structuralism generated by the existence of non-trivial automorphisms on certain structures. Section 4 offers a solution to the non-rigidity problem for this version of structuralism. The solution trades on removing the non-trivial automorphisms by introducing a collection of distinguished predicates into the languages that describe these structures. The concluding section 5 offers a philosophical justification for introducing these distinguished predicates in order to solve the problem of non-rigid structures.

\section{Mathematical Structuralism}

Structuralists argue that mathematics is not primarily about mathematical objects, like numbers, points, and sets. ${ }^{1}$ Mathematics is more concerned with the structures that these objects form, like the field of real numbers, the Euclidean plane, and the cumulative hierarchy of sets, as well as the mathematical theories that describe these structures. The theory of real analysis, for example, does not focus on the individual real numbers in isolation; real analysis focuses on the relations between real numbers, i.e., the structure that the real numbers exhibit.

Structuralist views can be roughly divided into two camps: eliminative and noneliminative. Non-eliminative structuralists argue that there is a single abstract structure that, e.g., real analysis is about. This is the structure exhibited by any complete ordered field, with the appropriate interpretation of the relations on this field. Real numbers are understood as positions in this unique abstract structure. Similarly, other mathematical theories concern other unique abstract structures, where the relevant mathematical objects are positions in those structures. These positions serve as the unique referents for singular mathematical terms. Eliminative structuralists, on the other hand, deny the existence of unique abstract structures, arguing that claims about the real number structure should be understood in terms of quantification over all complete ordered fields. This paper focuses on a particular version of non-eliminative structuralism.

Borrowing terminology from the literature on universals, some have applied the terms ante rem and in re to, respectively, the non-eliminative and eliminative characterizations of structuralism. However, we adopt a more fine-grained application of this terminology, which can be found in Shapiro $(2018, \S 1)$.

Any of the usual array of philosophical views on universals can be adapted to structures. One can be a Platonic ante rem realist, holding that each structure exists and has its properties independent of any systems that have that struc-

\footnotetext{
1 In what follows, I use the term 'structuralism' and its cognates to refer to mathematical structuralism.
} 
ture. On this view, structures exist objectively, and are ontologically prior to any systems that have them (or at least ontologically independent of such systems). Or one can be an Aristotelian in re realist, holding that structures exist, but insisting that they are ontologically posterior to the systems that instantiate them. Destroy all the natural number systems and, alas, you have destroyed the natural number structure itself. A third option is to deny that structures exist at all. Talk of structures is just a convenient shorthand for talk of systems that have a certain similarity.

On the fine-grained classification, ante rem and in re structuralism are both versions of non-eliminative structuralism, as both views hold that there exists some (abstract) entity that is, e.g., the structure of the reals. The difference between ante rem and in $r e$ structuralism lies in the relationship between an abstract structure and the realizations of that structure, i.e., the systems that exhibit the structure.

Consider the field of real numbers, which according to the non-eliminative structuralist is a unique abstract structure. This structure has multiple set-theoretic realizations, including the set of all equivalence classes of Cauchy sequences of rationals, and the set of Dedekind cuts of the rationals. ${ }^{2}$ Neither set is the abstract structure of the real number field. Rather, each set is a system or realization that exhibits this abstract structure.

The difference between ante rem and in re non-eliminative structuralism concerns the relationship between an abstract structure and the systems exhibiting that structure. The in re structuralist argues that the existence of an abstract structure depends, in some sense, on the existence of some system or other that exhibits the structure in question. The ante rem structuralist denies that any such dependence between structures and systems holds. ${ }^{3}$ One of the main tasks of non-eliminative structuralism is to articulate this dependence claim in order to make the distinction between ante rem and in re structuralism precise, as is discussed in Linnebo (2008) and Wigglesworth (2018).

Though the problem of non-rigid structures threatens both ante rem and in re structuralism, this paper addresses the problem only for a specific version of in $r e$ structuralism. According to this version of in re structuralism, which we call abstraction-based structuralism, abstract structures are obtained through a process of Fregean abstraction. This version of structuralism has recently been articulated and criticized by Linnebo and Pettigrew (2014). ${ }^{4}$ Their criticism specifically points to the fact that abstraction-based structuralism falls victim to the problem of

\footnotetext{
2 More precisely, the structure of the real number field is realized by systems that take one or the other of these sets as a domain, and include appropriate distinguished relations on the domain.

${ }^{3}$ For the ante rem structuralist, the existence of abstract structures is usually given axiomatically. See, e.g, Shapiro (1997) or Leitgeb (2014).

4 Linnebo and Pettigrew do not explicitly discuss this theory of abstract structures as an in re version of structuralism, though this is a natural interpretation based on the details of the theory. However, it is certainly a non-eliminative version of structuralism, and whether it is ante rem or in re is not particularly important for the remainder of the paper.
} 
non-rigid structures. By performing Fregean abstraction on a system of objects, we arrive at an abstract structure. But sometimes the abstraction process results in a mismatch between the system and the structure. The abstraction-based structuralist is forced to regard certain positions in a structure as identical, while the objects that correspond to those positions are distinct in the system from which the structure is abstracted. In the next section, we outline the details of Fregean abstraction and the abstraction-based structuralist position, following closely the account as given by Linnebo and Pettigrew. Section 3 develops the problem of non-rigid structures for abstraction-based structuralism, and Sect. 4 proposes a solution to this problem specifically for this version of structuralism.

\section{Abstraction-Based Structuralism}

Those familiar with contemporary philosophy of mathematics will recognize Fregean abstraction principles. Abstraction principles introduce identity conditions for abstract objects by appealing to equivalence relations. In this way Frege $(1968, \S 64)$ introduced identity conditions for the directions of lines by appealing to the equivalence relation of two lines being parallel: the direction of line $a$ is identical to the direction of line $b$ iff $a$ is parallel to $b$.

Identity conditions for abstract structures can also be given with a Fregean abstraction principle that appeals to an equivalence relation on systems of objects. Define a system $S=\left\langle D^{S}, R_{1}^{S}, \ldots, R_{n}^{S}\right\rangle$ to be an ordered tuple that includes a domain $D^{S}$ of objects and a number of distinguished relations $R_{1}^{S}, \ldots, R_{n}^{S}$ on the domain. The distinguished relations may include one-place relations, i.e., monadic properties. Systems may also include distinguished elements of the domain and distinguished functions on the domain. For simplicity, we focus on purely relational systems, though nothing turns on this restriction. An isomorphism between two systems is a relation-preserving bijective mapping between the objects in the domains of each system. ${ }^{5}$ The isomorphism relation between systems is an equivalence relation, so we can use it to provide identity conditions for abstract structures with a Fregean abstraction principle. Letting $[S]$ refer to the structure of system $S$, we have:

$$
[S]=\left[S^{\prime}\right] \leftrightarrow S \cong S^{\prime}
$$

That is, the structure of $S$ is identical to the structure of $S^{\prime}$ iff $S$ is isomorphic to $S^{\prime}{ }^{6}$ Given a system $S$, we say that $[S]$ is the pure structure of $S$, and that $S$ exhibits the structure $[S]$. It is desirable that $[S]$ be like $S$ in certain ways. In particular, it is desirable that, for all $S,[S] \cong S$. We will see, however, that abstraction-based structuralism does not allow for this isomorphism to hold in all cases. In particular,

\footnotetext{
5 This characterization of isomorphism will be made more precise in Sect. 4.

${ }^{6}$ Linnebo and Pettigrew (2014, p. 274) note that this abstraction principle can lead to the Burali-Forti paradox. As they suggest, one can avoid paradox by taking systems to be sets and abstractions to be sui generis mathematical objects.
} 
systems that admit non-trivial automorphisms may not be isomorphic to their own pure structures.

Given that we can compare systems with their pure structures, for instance to check whether the two are isomorphic, pure structures should also consist of a domain and distinguished relations on the domain. The domain contains elements, which we call 'positions' in the structure. The positions in the domain of a pure structure, and the relations between these positions, should in some sense match up with the objects and relations composing the systems that exhibit the pure structure in question. Like pure structures, positions in structures are also obtained through Fregean abstraction. When an object $x$ belongs to the domain of a system $S$, let $[x]_{S}$ be the corresponding position in $[S]$. Identity conditions for positions in structures are given by the following abstraction principle:

$$
[x]_{S}=\left[x^{\prime}\right]_{S^{\prime}} \leftrightarrow \exists f\left(f: S \cong S^{\prime} \wedge f(x)=x^{\prime}\right)
$$

Two positions in a structure are identical iff their corresponding objects are mapped to each other under an isomorphism. In particular, if $S=S^{\prime}$, then $f$ is an automorphism, and any two objects in $S$ that instantiate exactly the same distinguished relations can be mapped to the same position in the abstract structure [S]. This abstraction principle is what generates the non-rigidity problem, because there are systems that contain distinct objects satisfying all of the same distinguished relations in the system.

Despite being vulnerable to the non-rigidity problem, abstraction-based structuralism is a powerful theory of abstract structures. If one only focuses on rigid systems, such as the standard specifications of the systems of the natural numbers and the real numbers, then abstraction-based structuralism is an attractive and successful theory. In particular, it can prove that each rigid system has a unique pure structure, and that each system is isomorphic to its pure structure. It also allows for a principled distinction between structural and non-structural properties and relations. ${ }^{7}$ This success makes it a strong competitor to other versions of noneliminative structuralism, like Shapiro's ante rem structuralism. A solution to the problem of non-rigid structures for abstraction-based structuralism would make the theory even stronger.

\section{Non-rigid Structures}

Non-rigid structures, or in the present context non-rigid systems, are systems that admit at least one non-trivial automorphism, an isomorphism, $f$, from the system to itself that is not the identity mapping; for some $x$ in the domain of the system, $f(x) \neq x$. The problem that non-rigid systems present for abstraction-based structuralism can be seen by way of a simple example. Consider the following system, explicitly described by Linnebo and Pettigrew (2014, p. 276).

$$
G=\left\langle D^{G}=\{n, m\}, R^{G}=\{\langle n, m\rangle,\langle m, n\rangle\}\right\rangle
$$

\footnotetext{
7 See Linnebo and Pettigrew (2014, pp. 276-277), as well as Schiemer and Wigglesworth (2018).
} 
Fig. 1 System $G$

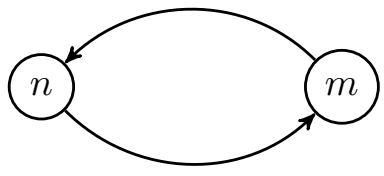

The system has two objects in its domain and a single symmetric and irreflexive distinguished relation that holds between them. The system can be visually represented as a simple graph with two nodes and two directed edges (Fig. 1).

In the system $G$ we have $R^{G}(n, m)$ and $R^{G}(m, n)$. There is a mapping $f$ from $G$ to itself such that $f(n)=m$ and $f(m)=n$. Because $R^{G}(n, m)$, we have $R^{G}(f(m), f(n))$; and similarly because $R^{G}(m, n)$, we have $R^{G}(f(n), f(m))$. It follows that $f: G \cong G$ is an automorphism. But $n \neq m$, so $f$ is non-trivial. The graph makes the existence of a non-trivial automorphism clear: permuting the two objects results in a graph with the same structure as the original.

In the system $G$ and its pure structure $[G]$, we see the non-rigidity problem concretely. The existence of the automorphism $f$ on the system $G$, when combined with the abstraction principle for positions in a structure, entails that the positions $[n]_{G}$ and $[m]_{G}$ in the pure structure $[G]$ are identical; but in the system $G$, the objects $n$ and $m$ are not identical. The mismatch between objects in $G$ and positions in $[G]$ further entails that $[G] \not \equiv G$.

The full force of the non-rigidity problem is apparent when considering more familiar mathematical systems that admit non-trivial automorphisms. The two node symmetric graph is not particularly noteworthy, mathematically speaking. But there are many non-rigid systems of mathematical interest. Standard examples discussed in the literature on mathematical structuralism include the complex field, under the automorphism that maps each complex number $a+b i$ to its conjugate $a-b i$. According to the abstraction principle for positions in structures, it follows that for all $a$ and $b, a+b i=a-b i$, and in particular $i=-i$. The integers considered as a group under addition has a non-trivial automorphism that maps each number $n$ to $-n$. If these cases weren't bad enough, consider the Euclidean plane. For any two points, $x, y$, in the Euclidean plane, there is an automorphism that maps $x$ to $y$. Abstraction-based structuralism would therefore collapse all of the positions in the structure of the Euclidean plane to a single point.

As a theory of structures, abstraction-based structuralism is simple, elegant, and highly successful when restricted to rigid systems. Unfortunately, as we have seen, it fails significantly for non-rigid structures. But the idea that pure mathematical structures are the result of abstracting away mathematically irrelevant properties from systems of objects is extremely natural and intuitive. Abstraction-based structuralism is a good first attempt at formally capturing this idea. It also represents a viable non-eliminative alternative to Shapiro's ante rem 
structuralism. For these reasons, a general solution to the non-rigidity problem for abstraction-based structuralism is highly desirable.

\section{A General Solution to the Non-rigidity Problem}

The general solution we propose focuses on the notions of identity and distinguishability of objects in a system. The solution has roots in some of the early 'abstractionist' approaches to mathematical structuralism. Consider, for instance, Dedekind's well-known characterization of the natural numbers in Was sind und was sollen die Zahlen?

If in the consideration of a simply infinite system $N$ set in order by a transformation $\phi$ we entirely neglect the special character of the elements; simply retaining their distinguishability ${ }^{8}$ and taking into account only the relations to one another in which they are placed by the order-setting transformation $\phi$, then are these elements called natural numbers or ordinal numbers or simply numbers, and the base-element 1 is called the base-number of the numberseries $N(1888$, Def. 73$)$.

Dedekind is here using abstraction to arrive at the pure structure of the natural numbers. In order for this abstraction to work, the objects in the system on which abstraction is performed must all be distinguishable from one another. The importance of the distinguishability of objects in a system is not unique to the natural numbers. The non-rigidity problem exploits an inability to distinguish between objects in particular systems. The solution to this problem should therefore focus on making those objects distinguishable. Once the objects in a system are distinguishable, abstraction on that system then results in the appropriate pure structure.

To present the solution, however, we need to be more precise in describing the problem. ${ }^{9}$ The problem of non-rigid systems requires that some systems admit nontrivial automorphisms. Recall that an automorphism is an isomorphism between a system and itself. An isomorphism, $f$, is a bijective mapping from the objects in the domain of one system to the objects in the domain of another that 'preserves relations'. So we must say what it means for an isomorphism to preserve relations. There is an intuitive notion of isomorphism between systems according to which $f$ is a mapping from the objects and distinguished relations of one system $S$ to, respectively, the objects and distinguished relations of another system $S^{\prime}$, such that for any objects, $x, y, \ldots$, and relation $R^{S}$, of system $S, R^{S}$ holds between $x, y, \ldots$ in $S$ if and only if $f\left(R^{S}\right)$ holds between $f(x), f(y), \ldots$ in $S^{\prime}$.

Unfortunately, this characterization of isomorphism is not precise enough to always determine whether or not an isomorphism holds between systems. Consider the following example, from

\footnotetext{
8 Emphasis added; subsequent emphases are original.

${ }^{9}$ Many thanks to an anonymous referee for helpful discussion of this point.
} 
Halvorson (2016, p. 593). Let $S=\left\langle D^{S}=\{a, b,\langle a, a\rangle\}, R^{S}=\{\langle a, a\rangle\}\right\rangle$, and let $S^{\prime}=\left\langle D^{S^{\prime}}=\{a, b,\langle a, b\rangle\}, R^{S^{\prime}}=\{\langle a, b\rangle\}\right\rangle$. The question of whether these systems are isomorphic is underdetermined. The answer depends on if the distinguished relations in these systems are one-place relations (in which case yes) or two-place relations (in which case no). Furthermore, both Halvorson (2013) and Glymour (2013) regard this characterization of isomorphism to be incoherent, because it allows elementarily inequivalent models to be isomorphic.

But the standard definition of isomorphism, familiar from model theory, is perfectly coherent. And because systems are similar to models, we can use the standard definition to determine whether $S$ and $S^{\prime}$ in the example above are isomorphic. This definition appeals to the notion of a formal language, or signature. Two models with the same signature are isomorphic when there is a bijection between the domains of the models that preserves 'corresponding' relations. Corresponding relations are relations that serve as the interpretation of the same relation symbol in the given signature. In Halvorson's example, $S$ and $S^{\prime}$ are then isomorphic if $R^{S}$ and $R^{S^{\prime}}$ serve as the interpretation of a one-place predicate symbol in the relevant signature. If, on the other hand, they serve as the interpretation of a two-place predicate symbol, then the systems are not isomorphic.

According to the model-theoretic definition, when an isomorphism under consideration is an automorphism, the interpretation of the relations (and of the other symbols in the signature) remains fixed. In other words, when the isomorphism is an automorphism, corresponding relations are identical. The problem of non-rigid systems, which turns on the existence of non-trivial automorphisms on a system, can be recast in this model-theoretic framework.

To make the notion of isomorphism precise, let $\mathscr{L}$ be a signature with a set of primitive relation symbols. ${ }^{10}$ An $\mathscr{L}$-system is an interpretation $\mathcal{I}=\left\langle D^{\mathcal{I}}, v^{\mathcal{I}}\right\rangle$, comprising a domain and an assignment function that maps each $m$-place relation symbol, $R$, to a set $R^{I}$ of $m$-tuples of elements of the domain. Given a signature $\mathscr{L}$, an isomorphism is a relation between $\mathscr{L}$-systems. Two $\mathscr{L}$-systems, $\mathcal{I}=\left\langle D^{\mathcal{I}}, v^{\mathcal{I}}\right\rangle$ and $\mathcal{J}=\left\langle D^{\mathcal{J}}, v^{\mathcal{J}}\right\rangle$ are isomorphic iff there is a bijection $f: D^{\mathcal{I}} \rightarrow D^{\mathcal{J}}$ such that, for all $x_{1}, \ldots, x_{m} \in D^{\mathcal{I}}$, if $R$ is an $m$-place relation symbol, $\left\langle x_{1}, \ldots, x_{m}\right\rangle \in R^{I}$ iff $\left\langle f\left(x_{1}\right), \ldots, f\left(x_{m}\right)\right\rangle \in R^{\mathcal{J}}$. The isomorphism is an automorphism when $\mathcal{I}=\mathcal{J}$.

According to this definition, the relation of being isomorphic is something that holds between $\mathscr{L}$-systems (as opposed to systems). According to abstraction-based structuralism, identity conditions for pure structures are given to us through an abstraction principle, which involves the isomorphism relation on the right hand side. Given the difficulties with the notion of isomorphism as it applies to systems, it is more appropriate that, for abstraction-based structuralism, pure structures should be understood as being abstracted from $\mathscr{L}$-systems: two $\mathscr{L}$-systems have the same pure structure if and only if they are isomorphic.

$$
[\mathcal{I}]=[\mathcal{J}] \leftrightarrow \mathcal{I} \cong \mathcal{J}
$$

\footnotetext{
${ }^{10}$ What follows are standard model-theoretic definitions. As before, we consider relational $\mathscr{L}$-systems, though one could also use interpretations to assign values to constants and function symbols in a signature.
} 
Recall that abstraction-based structuralism appeals to a second abstraction principle to determine the identity conditions for positions in pure structures. Given some element $x \in D^{I}$, let $[x]_{\mathcal{L}}$ be the corresponding position in $[\mathcal{I}]$. Two objects in $\mathscr{L}$-systems have the same position when there is an isomorphism between the systems that maps one to the other.

$$
[x]_{\mathcal{I}}=[y]_{\mathcal{J}} \leftrightarrow \exists f(f: \mathcal{I} \cong \mathcal{J} \wedge f(x)=y)
$$

Understanding isomorphism as a relation between $\mathscr{L}$-systems, and using the standard, model-theoretic definition of isomorphism, one can restate the problem of non-rigid systems in these terms. Starting with the two node symmetric graph $G=\left\langle D^{G}=\{n, m\}, R^{G}=\{\langle n, m\rangle,\langle m, n\rangle\}\right\rangle$, consider the $\mathscr{L}$-system $\mathcal{G}=\left\langle D^{G}, v^{\mathcal{G}}\right\rangle$ that maps the binary relation symbol $R \in \mathscr{L}$ to the set of ordered pairs $R^{G}$. Under the mapping $f: D^{G} \rightarrow D^{G}$, such that $f(n)=m$ and $f(m)=n$, we see that for all $x, y \in D^{G},\langle x, y\rangle \in R^{G}$ iff $\langle f(x), f(y)\rangle \in R^{G}$. So $f$ is an automorphism, and it is nontrivial, as $n \neq m$.

The solution to the problem of non-rigid systems, or more precisely non-rigid $\mathscr{L}$ -systems, focuses on preserving the distinguishability of the objects in the $\mathscr{L}$-systems that serve as the basis for abstraction. This kind of solution has been explored by some who are interested in the non-rigidity problem as it applies to ante rem structuralism. Leitgeb and Ladyman (2008) suggest that the identity and non-identity relations can be included as distinguished relations in the specification of a system.

We conclude that the identity relation for positions in a structure is a relation that ought to be viewed as an integral component of a structure in the same way as, for example, the successor relation is an integral component of the structure of natural numbers (p. 390).

Adapting the structuralist slogan on natural numbers ... we are still allowed to say that "There is no more to the individual [positions] "in themselves" than the relations they bear to each other', the only addition that we have to make is that we have to count identity and difference of [positions] among the very relations that the [positions] in a [structure] bear to each other (pp. 392-393).

Leitgeb and Ladyman argue that the identity or difference of positions in a structure should be given by the structure itself, and so the relations of identity and non-identity should be included as essential parts of a system.

Their proposal suggests an approach to the non-rigidity problem for abstraction-based structuralism, a possible strategy to rigidify non-rigid $\mathscr{L}$-systems. If one were dealing with systems, as opposed to $\mathscr{L}$-systems, one could adopt Leitgeb and Ladyman's approach by expanding a system $S=\left\langle D^{S}, R_{1}^{S}, \ldots, R_{n}^{S}\right\rangle$ to a system $S^{\prime}=\left\langle D^{S}, R_{1}^{S}, \ldots, R_{n}^{S}, I^{S}, N^{S}\right\rangle$, which includes the identity and non-identity relations on the domain. Such an expansion would make these relations distinguished, if they were not distinguished already. One could then abstract on the expanded system $S^{\prime}$ to obtain the pure structure of $S$. It isn't hard to see, however, that adding the identity and non-identity relations will not remove any non-trivial automorphisms. Both 
relations are symmetric, and so any non-trivial automorphism on $S$ is a non-trivial automorphism on $S^{\prime}$, as the relations would not enable one to distinguish between previously indistinguishable objects.

Adapting this approach for $\mathscr{L}$-systems, one would expand a signature with new distinguished predicates, rather than expanding a system with new distinguished relations. But identity and non-identity are usually already included in the logical part of the languages used to describe $\mathscr{L}$-systems. So any $\mathscr{L}$-systems that admit non-trivial automorphisms do so despite the identity and non-identity relations being distinguished. If for some reason identity and non-identity are not included in the logical part of a language used to describe an $\mathscr{L}$-systems, adding them will not help. As before, the relations are symmetric, so they do not allow one to make new distinctions between objects that were previously indistinguishable.

Focusing on the identity and non-identity relations, as Leitgeb and Ladyman suggest, does not yield a solution to the non-rigidity problem for abstraction-based structuralism. However, there is another way to ensure that all objects in an $\mathscr{L}$-system are distinguishable. If one were dealing with systems, as opposed to $\mathscr{L}$-systems, one could expand a system $S=\left\langle D^{S}, R_{1}^{S}, \ldots, R_{n}^{S}\right\rangle \quad$ to a system $S^{*}=\left\langle D^{S}, R_{1}^{S}, \ldots, R_{n}^{S}, P_{x_{1}}^{S}, \ldots, P_{x_{m}}^{S}\right\rangle$, which includes a one-place relation (i.e., a property) for each distinct object in the domain. As we are working in the context of $\mathscr{L}$ -systems, however, the analogous approach adds one-place relation symbols, $P_{x_{1}}, \ldots, P_{x_{m}}$, to the relevant signature. These one-place predicates can then be interpreted by the singleton sets of each object in the relevant domain.

For example, recall the $\mathscr{L}$-system $\mathcal{G}=\left\langle D^{G}, v^{\mathcal{G}}\right\rangle$, which represents the twonode symmetric graph, so that $D^{G}=\{n, m\}$, and the assignment function $v^{\mathcal{G}}$ maps the non-logical binary relation symbol $R \in \mathscr{L}$ to the set of ordered pairs $R^{G}=\{\langle n, m\rangle,\langle m, n\rangle\}$. This $\mathscr{L}$-system admits a non-trivial automorphism $f$ that permutes $n$ and $m$. To remove the non-trivial automorphism on the $\mathscr{L}$-system $\mathcal{G}$, let $\mathcal{G}^{*}=\left\langle D^{G}, v^{\mathcal{G}^{*}}\right\rangle$ be an $\mathscr{L}^{*}$-system, where $\mathscr{L}^{*} \supseteq \mathscr{L}$ adds one-place relation symbols $P_{n}$ and $P_{m}$ to $\mathscr{L}$, one relation symbol for each element of $D^{G}$. The $\mathscr{L}^{*}$-system $\mathcal{G}^{*}$ is a signature expansion of the $\mathscr{L}$-system $\mathcal{G}$. The assignment function $v^{\mathcal{G}^{*}}$ maps the two-place predicate $R$ as before, and maps the new one-place predicates, $P_{n}$ and $P_{m}$, to $\{n\}$ and $\{m\}$, respectively. The mapping, $f$, which permutes $n$ and $m$, is no longer an automorphism, because the biconditionals $n \in\{n\}$ iff $f(n) \in\{n\}$ and $m \in\{m\}$ iff $f(m) \in\{m\}$ do not hold. In fact, there are no non-trivial automorphisms on the expanded $\mathscr{L}^{*}$-system $\mathcal{G}^{*}$; the only automorphism on this $\mathscr{L}^{*}$-system is the identity mapping.

Generally, all that is required for this solution to work is that for every distinct object, $x$, in the domain of an $\mathscr{L}$-system $S$, there is a one-place relation symbol $P_{x}$ in the signature of $\mathscr{L}^{*}$-system $S^{*}$, where $S^{*}$ is a signature expansion of $S$. Call these predicates identity predicates, as the assignment function $v^{S^{*}}$ maps each $P_{x}$ to $\{x\}$, which is extensionally identical to the property being identical to $x$. Call a signature expansion of an $\mathscr{L}$-system that (only) adds identity predicates to the signature, mapping each new identity predicate to the appropriate singleton set, an identity expansion. 
Once an $\mathscr{L}$-system $S$ is expanded to an $\mathscr{L}^{*}$-system $S^{*}$, all objects in the $\mathscr{L}^{*}$-system are distinguishable because each object can be picked out by its unique identity predicate, a predicate that isn't satisfied by any other object. In this way, the proposal allows for a solution to the non-rigidity problem for abstraction-based structuralism, when the relevant abstraction principles are applied to $\mathscr{L}$-systems, rather than systems detached from a signature.

One might worry that this introduces a 'language relativity' into the notion of pure mathematical structure. However, it seems that this appeal to language may be required, given the arguable incoherence, or at least indeterminacy, of the intuitive, language-independent, notion of isomorphism. Furthermore, mathematical structures are generally understood and explored in the context of a mathematical theory, given in terms of a specified signature. For example, we think of the natural number structure, and the relations between natural numbers such as 'less than', in terms of the signature, or language, of Peano arithmetic. For purposes of this paper, then, we assume that pure mathematical structures are characterized relative to a given signature.

There is an alternative presentation of this solution, which captures the main idea in a slightly different way. Instead of expanding an $\mathscr{L}$-system with one-place predicate symbols, we could expand it with constants, one for each object in the domain. As long as identity is included in the logical part of the relevant language, both presentations solve the abstraction-based structuralist's non-rigidity problem. One might think that whether one presentation is preferable to the other boils down to how one feels, in the spirit of Quine (1951), about ontology versus ideology. One can add more objects or add more relations to the specification of a system. But it is important to keep in mind for the present context that in neither case is anything really being added. Any new constant refers to an object that is already in the $\mathscr{L}$-system's domain, and has been all along. And any new predicate refers to a relation that holds whether we specify it or not. The only thing that is changing is the language used to describe the system.

It should be noted that neither presentation of this solution solves all of the abstraction-based structuralist's problems. In discussing models of complex analysis, Shapiro (2012, p. 383) notes that adding a constant symbol $i$ (or equivalently, an identity predicate that only $i$ satisfies) to the language of complex analysis, with the axiom $i^{2}=-1$, makes the system rigid. Adding the constant $i$ entails that there will be a distinguished object that is the referent of $i$. Though this addition makes the system rigid, it does not determine which of the two square roots of -1 the constant $i$ refers to. The system where $i$ refers to one square root and not the other is equivalent to the system that switches the referents around. In this sense the two square roots of -1 are indistinguishable. While this is an issue the abstraction-based structuralist may be concerned with, especially when considering the relationship between the language of complex analysis and the system of complex numbers, it is not the specific problem that we are attempting to solve here. After all, adding a constant symbol, or an identity predicate, makes the model rigid. And this is our primary concern.

With the proposed solution in hand, for each $\mathscr{L}$-system $S$, there is an expanded $\mathscr{L}^{*}$-system $S^{*}$ that gives rise to the intended pure structure of $S$ through Fregean abstraction. For the abstraction-based structuralist, the process of Fregean 
abstraction is perhaps best understood as a two-step process. The first step takes one from an $\mathscr{L}$-system $S$ to the expanded $\mathscr{L}^{*}$-system $S^{*}$. It may be that $\mathscr{L}$ already includes identity predicates for all of the objects in the domain of $\mathcal{S}$. If $v^{S}$ maps these identity predicates in the appropriate way, then $S=S^{*}$. But usually $S^{*}$ is a proper signature expansion of $S$. After the first step of moving to an expanded $\mathscr{L}^{*}$ -system, the second step uses abstraction to obtain $\left[\mathcal{S}^{*}\right]$, which is the intended pure structure of $S$.

Arguably, abstraction on expanded $\mathscr{L}^{*}$-systems is a plausible rational reconstruction of mathematical practice. First fix the identity and distinctness of objects in the relevant $\mathscr{L}$-system by expanding it to an $\mathscr{L}^{*}$-system, then use Fregean abstraction to obtain the pure structure of that $\mathscr{L}^{*}$-system. When analysts talk about the structure of the complex numbers, they know what the target is. They are talking about a structure where $i$ and $-i$ are distinguishable. There are certainly pure structures, obtained through Fregean abstraction on certain $\mathscr{L}$-systems, that identify (what those systems call) $i$ with $-i$. But these structures are not the intended pure structure of the complex numbers. If the structure of the complex numbers is to be obtained through Fregean abstraction on an $\mathscr{L}$-system, then the distinguishability of $i$ and $-i$ must be given by the $\mathscr{L}$-system already, so that it can then be preserved in the pure structure.

So this solution to the problem of non-rigid structures is a possible description of how intended mathematical structures are in practice (perhaps implicitly) obtained through Fregean abstraction. It may not be the only solution. It would be nice for the abstraction-based structuralist to have some way of avoiding the non-rigidity problem without inflating the signatures of $\mathscr{L}$-systems to include identity predicates for all of the objects in its domain. Whether this is possible remains to be seen. But at least non-eliminative structuralists appealing to Fregean abstraction have one solution to the problem of non-rigid structures. The important question is whether this solution is philosophically acceptable. The next section argues that it is.

\section{The Philosophical Acceptability of Identity Predicates}

What justifies adding individual identity predicates to the signature of an $\mathscr{L}$-system? That depends, in part, on what justifies adding any predicate to the signature of an $\mathscr{L}$-system. Generally, relation symbols in a signature $\mathscr{L}$ are supposed to pick out relations on the domain of $\mathscr{L}$-systems. But there are no specific restrictions on the relations that these predicates can refer to, other than that they should match in arity, so that one-place relation symbols pick out one-place relations (singletons), twoplace relation symbols pick out two-place relations (ordered pairs), etc. Given any $\mathscr{L}$-system $S$, there doesn't seem to be anything stopping us from adding an identity predicate to $\mathscr{L}$ for each object in $D^{S}$. An identity predicate $P_{x}$ picks out a property, which we may call the identity property, being identical to $x$, or at least one that is extensionally identical to that property. 
However, our ability to pinpoint an identity expansion of an $\mathscr{L}$-system, in order to arrive at that $\mathscr{L}$-system's pure structure through abstraction, raises more general questions about whether and how we can choose which $\mathscr{L}$-system to abstract from. ${ }^{11}$ This question is particularly relevant when one is interested in finding the pure structure described by a particular mathematical theory, like number theory or complex analysis. The abstraction-based structuralist argues that mathematical theories describe pure structures. These structures are obtained through a process of abstraction on particular $\mathscr{L}$-systems, where $\mathscr{L}$ is the language of the relevant theory. But whether or not we arrive at the appropriate pure structure for the theory in question depends on the $\mathscr{L}$-system that serves as the basis for abstraction.

Unfortunately, for a given theory, there are often many systems to choose from. $\mathscr{L}$-systems are models, and in a first-order setting, many theories have a large number of models that are not isomorphic to one another. The existence of these models follows from the (upward) Löwenheim-Skolem theorem, which says that every countable first-order theory with an infinite model has a model of every infinite size. For example, the first-order theory of the natural numbers has a model of every infinite size, because it has a model — the standard model—which is countably infinite. Abstracting on these different models of number theory will lead to pure structures that are not isomorphic to one another. So how do we choose which $\mathscr{L}$-system to abstract from in order to ensure that we arrive at the structure of the natural numbers, the intended pure structure that number theory describes? Similar questions arise for other mathematical theories of interest, so we need a general strategy for making this choice.

A plausible strategy, in the first-order setting, may be to choose the standard model of the theory in question. ${ }^{12}$ If we are able to choose the basis for abstraction, surely the standard model of a theory will lead us to the appropriate pure structure that the theory describes. This strategy is reasonable, because for many mathematical theories of interest, the standard model of the theory is rigid. This holds for the standard model of number theory, as well as for the standard model of real analysis. Choosing either of these $\mathscr{L}$-systems as the basis for abstraction results in the appropriate pure structure of the theory in question.

But not all standard models are rigid. The standard model for complex analysis is not. Performing abstraction on this non-rigid $\mathscr{L}$-system yields a pure structure that is not isomorphic to the original $\mathscr{L}$-system, as it identifies each complex number with its conjugate. So choosing the standard model of a mathematical theory as the basis for abstraction does not always work. It cannot be the general strategy for choosing $\mathscr{L}$-systems to abstract from, because it won't always deliver the pure structure of the theory in question.

But it is a good start. Focusing on the standard model of a theory, the abstraction-based structuralist can use considerations from Sect. 4 to find a rigid, expanded $\mathscr{L}^{*}$-system able to serve as the basis for abstraction in order to arrive at the pure structure of the theory in question. In the case of complex analysis, start with the

11 Thanks to an anonymous referee for discussion of this point.

12 For the second-order case, if the theory in question is categorical, then any model will do. 
theory's standard model, which is non-rigid, and expand the signature of the theory with identity predicates for each object in the domain, ensuring that the valuation function of the expanded $\mathscr{L}^{*}$-system maps each identity predicate appropriately. Abstracting on the expanded $\mathscr{L}^{*}$-system results in the pure structure of the complex numbers. The strategy can be generalized, because for any particular $\mathscr{L}$-system, one can expand $\mathscr{L}$ with identity predicates, one for each object in the system's domain. Abstracting on the expanded $\mathscr{L}^{*}$-system results in the pure structure of the original $\mathscr{L}$-system. If one is interested in the pure structure of a particular mathematical theory, start with the standard model of the theory (or an $\mathscr{L}$-system that is isomorphic to the standard model) and expand the signature with identity predicates for each object in the domain. Once the model has been expanded, abstract on the expanded model to arrive at the intended pure structure of the theory in question.

Because this general strategy appeals to identity predicates, which refer to identity properties, it is appropriate to address one further concern as to whether these properties are controversial on metaphysical grounds. Identity properties are very similar to other kinds of properties known as haecceities. Haecceities, like identity properties, are also properties that are instantiated by exactly one object. Let $H_{x}^{S}$ represent the haecceity of an object $x$ in $\mathscr{L}$-system $S$. Because of the nature of haecceities, it follows that for all $y, y \in H_{x}^{S} \leftrightarrow y=x$. Of course, this biconditional also holds for $x$ 's identity property. What this shows is that identity properties and haecceities have the same extension. But it does not show that they are the same thing.

Haecceities are usually connected to more controversial metaphysical notions like essences or thisnesses. We are not arguing that any of these metaphysical connections hold for identity properties. The identity property of an object is simply a property that it and it alone possesses, nothing more. So identity properties shouldn't be controversial for any similarities they may bear to haecceities. But they shouldn't be particularly controversial for any other metaphysical reason. Pure structures are obtained through Fregean abstraction on $\mathscr{L}$-systems, and $\mathscr{L}$-systems are understood set-theoretically. Set-theoretically, identity properties are basic and quite unproblematic; they are simply singletons of the elements of the domain.

Since there do not appear to be any specific criteria that predicates must satisfy in order to be added to the signature of an $\mathscr{L}$-system, identity predicates are fair game. If these predicates are included, and mapped appropriately to identity properties, then the expanded $\mathscr{L}^{*}$-systems do not admit non-trivial automorphisms. Thus we have a general solution to the problem of non-rigid structures for abstraction-based structuralism, one that can be applied to any $\mathscr{L}$-system of objects or any theory that the mathematician is interested in.

Acknowledgements Open access funding provided by University of Vienna. This project has received funding from the European Research Council (ERC) under the European Union's Horizon 2020 research and innovation programme (Grant Agreement No. 715222). Prior to this, research leading to this paper was carried out within the ANRDFG project "Mathematics: Objectivity by Representation" (Grant No. LE 2500/4-1). I wish to thank Hannes Leitgeb, Øystein Linnebo, Gil Sagi, Georg Schiemer, and several anonymous referees for helpful comments and discussions of this material.

Open Access This article is distributed under the terms of the Creative Commons Attribution 4.0 International License (http://creativecommons.org/licenses/by/4.0/), which permits unrestricted use, distribution, 
and reproduction in any medium, provided you give appropriate credit to the original author(s) and the source, provide a link to the Creative Commons license, and indicate if changes were made.

\section{References}

Burgess, J. (1999). Review of Shapiro (1997). Notre Dame Journal of Formal Logic, 40, 283-291.

Dedekind, R. (1888). Essays on the theory of numbers (W. W. Beman, Trans.). Chicago: Open Court.

Frege, G. (1968). The foundations of arithmetic (J. L. Austin, Trans.). Oxford: Basil Blackwell.

Glymour, C. (2013). Theoretical equivalence and the semantic view of theories. Philosophy of Science, $80,286-297$.

Halvorson, H. (2013). The semantic view, if plausible, is syntactic. Philosophy of Science, 80, 475-478.

Halvorson, H. (2016). Scientific theories. In P. Humphreys (Ed.), Oxford handbook of philosophy of science (pp. 585-608). Oxford: Oxford University Press.

Hellman, G. (2001). Three varieties of mathematical structuralism. Philosophia Mathematica, 9, $184-211$.

Keränen, J. (2001). The identity problem for realist structuralism. Philosophia Mathematica, 9, 308-330.

Keränen, J. (2006). The identity problem for realist structuralism II: A reply to Shapiro. In F. MacBride (Ed.), Identity and modality (pp. 146-163). Oxford: Clarendon Press.

Leitgeb, H. (2014). On mathematical structuralism. Paper presented at first symposium on the foundations of mathematics, Vienna.

Leitgeb, H., \& Ladyman, J. (2008). Criteria of identity and structuralist ontology. Philosophia Mathematica, 16, 388-396.

Linnebo, Ø. (2008). Structuralism and the notion of dependence. Philosophical Quarterly, 58, 59-79.

Linnebo, Ø., \& Pettigrew, R. (2014). Two types of abstraction for structuralism. Philosophical Quarterly, $64,267-283$.

Quine, W. V. O. (1951). Ontology and ideology. Philosophical Studies, 2, 11-15.

Schiemer, G., \& Wigglesworth, J. (2018). The structuralist thesis reconsidered. The British Journal for the Philosophy of Science. https://doi.org/10.1093/bjps/axy004.

Shapiro, S. (1997). Philosophy of mathematics: Structure and ontology. Oxford: Oxford University Press.

Shapiro, S. (2012). An " $i$ " for an $i$ : Singular terms, uniqueness, and reference. Philosophia Mathematica, $5,380-415$.

Shapiro, S. (2018). Mathematical structuralism. The Internet Encyclopedia of Philosophy, ISSN 21610002. http://www.iep.utm.edu/m-struct/. Accessed 20 September 2018.

Wigglesworth, J. (2018). Grounding in mathematical structuralism. In R. Bliss \& G. Priest (Eds.), Reality and its structure: Essays in fundamentality (pp. 218-236). Oxford: Oxford University Press. 